\title{
Influence of Endometrial Microbiota on Reproductive Outcomes in IVF Programs
}

\author{
Veronika Smolnikova ${ }^{1 *}$, Lela Keburiya ${ }^{1}$, Tatiana Priputnevich ${ }^{1}$, Vera Muravieva ${ }^{1}$, Elena \\ Kalinina ${ }^{1}$ and Gennadiy Sukhikh ${ }^{1}$ \\ ${ }^{1}$ Department of Gynecology and Perinatology, Kulakovs Scientific Centre of Obstetrics, Russia
}

*Corresponding author: Veronika Smolnikova, Kulakovs Scientific Centre of Obstetrics, Gynecology and Perinatology, Moscow, Russia.

To Cite This Article: Veronika Smolnikova. Influence of Endometrial Microbiota on Reproductive Outcomes in IVF Programs. Am J Biomed Sci \& Res. 2019 - 4(3). AJBSR.MS.ID.000797. DOI: 10.34297/AJBSR.2019.04.000797

Received: July 24, 2019 | Published: July 30, 2019

\begin{abstract}
Introduction: Implantation failures are one of the causes of the inefficiency of IVF programs. Recent studies have shown that in humans, some biotopes such as the uterus and placenta, previously considered sterile, are colonized by their unique microbiota. Disturbance of the uterine microbiota can be one of the reasons for the decline in the effectiveness of assisted reproductive technology programs.

Materials and methods: A total of 80 patients with infertility were enrolled in the study: group I included the patients with fresh embryo transfer and group II included the patients with frozen-thawed embryo transfer. After routine embryo transfer, the most distal $5 \mathrm{~mm}$ portion of the embryo transfer catheter was placed in the sterile container. A study was conducted using culture-based methods with extended nutrient media.

Results: In patients with fresh embryo transfer in ovarian hyperstimulation cycle, 28 species of microorganisms were detected. Pregnancy in this group occurred in 20 women ( $40 \%$ ). The microflora was dominated by Lactobacillus spp. ( $90 \%$ of women). In patients with frozen-thawed embryo transfer, 20 species of microorganisms were detected. Pregnancy in this group occurred in 13 women (43.3\%). The microflora was dominated by Lactobacillus spp. ( $69.2 \%$ of women). Bifidobacterium spp. were significantly more frequently detected in patients of group II than in those of group I ( $23.3 \%$ and $2 \%$, respectively).
\end{abstract}

Conclusion: The evidence of this study suggests that the uterus is not sterile, the uterine microbiota may differ from the cervical canal microbiota, the uterine microbiota is one of the predictors of the success of embryo implantation.

Keywords: Infertility; Endometrium; Uterine microbiota; Embryo transfer

\section{Introduction}

Implantation failures are one of the causes of the inefficiency of IVF programs. Recent studies have shown that in humans, some biotopes such as the uterus and placenta, previously considered sterile, are colonized by their unique microbiota. Disturbance of the uterine microbiota can be one of the reasons for the decline in the effectiveness of assisted reproductive technology programs [1-4]. It is known that microorganisms affect the immune system - Accordingly, the effect of microorganisms on the immune environment before pregnancy is associated with the endometrial receptivepotential[5,6]. Successfulembryoimplantation dependson endometrial condition at the moment of the "implantation window". Endometrial susceptibility deterioration during the menstrual cycle leads to impaired placentation and infertility [7]. At the same time, the commensal microbiota can provide the protection against pathogenic microorganisms and contribute to the maintenance of homeostasis in the uterine cavity $[7,8]$. The purpose of our research is to evaluate the effect of uterine microbiota on the success of embryo implantation in women in assisted reproductive technology programs.

\section{Materials and Methods}

A total of 80 patients with infertility were enrolled in the study: group I included the patients with fresh embryo transfer and group II included the patients with frozen-thawed embryo transfer. The average age of women was \pm 31.4 years. After routine embryo transfer, the most distal $5 \mathrm{~mm}$ portion of the embryo transfer catheter was placed in the sterile container. A study was conducted using culture-based methods with extended nutrient media. In patients with implantation failures in history, 16S RNA sequencing is planned. In order to exclude other negative factors and to make 
more accurate assessment of the role of microbiota in reproductive outcomes, we included women who were transferred embryos of good and excellent quality both in fresh embryo transfer and frozen-thawed embryo transfer.

\section{Results}

It was established that the uterine cavity in women of groups I and II was sterile in $12.0 \%$ and $13.3 \%$ of cases, respectively. The selected microflora in the comparison groups was characterized by the presence of monocultures (28\% of women in group I and $30 \%$ in group II) and associations of microorganisms (52.9\% and 56.6\%, respectively). The most represented species in the microbiota were Lactobacillus, their isolation frequency was $80 \%$ и $66.6 \%$. Moreover, these microorganisms were present in the monoculture in $26 \%$ of patients in group I and in $20 \%$ in group II; they were detected in association with each other in $14 \%$ and $13.3 \%$, and in association with opportunistic microorganisms in $38 \%$ and $20 \%$ of cases, respectively. The opportunistic microorganisms were the second most frequent component of the microbiota in $48 \%$ of women in group I and in $40 \%$ of women in group II. In the monoculture, opportunistic microorganisms were isolated in $2 \%$ and $3.3 \%$ of women, associations of only opportunistic microorganisms were revealed in $8 \%$ and $6.6 \%$ of women, associations of opportunistic microorganisms with Lactobacillus spp. and Bifidobacterium spp. were detected in $38 \%$ and $29.9 \%$ of women, respectively. Bifidobacterium spp. were significantly more frequently detected in patients of group II than in those of group I (23.3\% and $2 \%$, respectively).

In patients with fresh embryo transfer in ovarian hyperstimulation cycle, 28 species of microorganisms were detected (Table 1). Pregnancy in this group occurred in 20 women ( $40 \%$ ). The microflora was dominated by Lactobacillus spp. (90\% of women): L. jensenii (40\%), L. crispatus (35\%). The most common opportunistic microorganisms (50\%) were Gardnerella vaginalis (G. vaginalis) and Enterococcus faecalis (E. faecalis) (20\%), Streptococcus anginosus (S. anginosus) (15\%). In non-pregnant women (60 \%) the frequency of isolation of Lactobacillus spp. was lower than in pregnant women (73.3\%), but L. jensinii $(53.3 \%)$ and L. crispatus (26.6\%) were dominating. Among opportunistic microorganisms, G. vaginalis (10\%) and optional anaerobes S. anginosus (10\%) and Staphylococcus haemolyticus (6.6\%) were most frequently detected. It should be noted that Haemophilus imfluenzae (3.3\%) and Actinomyces sp. (6.6\%) which may be seldom found in the reproductive organs were also revealed.

Table 1: Detected species of microorganisms.

\begin{tabular}{|c|c|}
\hline Patients with fresh embryo transfer, $n=50$ & Patients with frozen-thawed embryo transfer, $n=30$ \\
\hline Lactobacillus spp.: & Lactobacillus spp.: \\
\hline 1. Lactobacillus jensenii & 1. Lactobacillus jensenii \\
\hline 2. L.crispatus & 2. L.crispatus \\
\hline 3. L. gasseri & 3. L. gasseri \\
\hline 4. L. oris & 4. L.iners \\
\hline 5. L. vaginalis & 5. L. vaginalis \\
\hline 6. L. johnsonii & 6. L. fermentum \\
\hline \multirow[t]{2}{*}{ 7. L. reuteri } & 7. L. reuteri \\
\hline & 8. L. paracasei \\
\hline Opportunistic microorganisms: & Opportunistic microorganisms: \\
\hline 8. Escherichia coli & 9. Escherichia coli \\
\hline 9. Proteus mirabilis & 10. Enterococcus faecalis \\
\hline 10.Staphylococcus epidermidis & 11. Staphylococcus epidermidis \\
\hline 11. S. hominis & 12. S. warneri \\
\hline 12. S. capitis & 13. Streptococcus agalactiae \\
\hline 13. S. haemolyticus & 14. $\underline{\text { Sanginosus }}$ \\
\hline 14. Enterococcus faecalis & 15. Actinomyces neuii \\
\hline 15. Streptococcus agalactiae & 16. Micrococcus luteus \\
\hline 16. S. salivarius & 17. Propionibacterium avidum \\
\hline \multicolumn{2}{|l|}{ 17. S.anginosus } \\
\hline \multicolumn{2}{|l|}{ 18. Micrococcus luteus } \\
\hline \multicolumn{2}{|l|}{ 19. Haemophilus influenzae } \\
\hline \multicolumn{2}{|l|}{ 20. Atopobuim vaginae } \\
\hline \multicolumn{2}{|l|}{ 21. Veillonella atypica } \\
\hline 22. Actinomyces radingae & \\
\hline
\end{tabular}




\begin{tabular}{|c|c|}
\hline 23. Actinomyces neuii & \\
\hline 24. Dialister micraerophilus & \\
\hline 25. Propionibacterium avidum & \\
\hline 26. P. acnes & \\
\hline 27. Gardnerella vaginalis & Bifidobacterium spp. \\
\hline Bifidobacterium spp. & 18. Bifidobacterium bifidum \\
\hline 28. Bifidobacterium bifidum & 19. B. dentium \\
\hline & 20. B. longum \\
\hline
\end{tabular}

In patients with frozen-thawed embryo transfer, 20 species of microorganisms were detected (Table 1). Pregnancy in this group occurred in 13 women (43.3\%). The microflora was dominated by Lactobacillus spp. (69.2\% of women): L. jensenii (46.2\%), L. crispatus (23\%). The most common opportunistic microorganisms (38.5 \%) were optional anaerobes Escherichia coli (15.4\%) and Staphylococcus epidermidis (S. epidermidis) (15.4\%). In non-pregnant women $(56.7 \%)$ the frequency of isolation of Lactobacillus spp. was lower than in pregnant women (64.7\%); L. jensinii (41.2\%) and L. crispatus (23.5\%) were dominating. Among opportunistic microorganisms, optional anaerobes S. epidermidis $(17.6 \%)$ and E. faecalis (11.8\%) were most frequently detected. However, Bifidobacterium spp. were detected in 35.3\% of women, and Actinomyces neuii were found in $5.9 \%$ of women.

\section{Discussion}

The results of the current study are consistent with those of M. Mitchell and E. Cicinelli who suggested that the uterine cavity is not sterile $[9,10]$. Moreno I. et al. performed the analysis of the microbiota in paired samples of endometrial fluid and vaginal aspirates obtained simultaneously from 13 fertile woman in prereceptive and receptive phases within the same menstrual cycle (a total of 52 samples were analyzed). To investigate the hormonal regulation of the endometrial microbiota during the acquisition of endometrial receptivity, endometrial fluid was collected at prereceptive and receptive phases within the same cycle from 22 fertile women (a total of 44 samples were analyzed). Finally, the reproductive impact of an altered endometrial microbiota in endometrial fluid was assessed by implantation, ongoing pregnancy, and live birth rates in 35 infertile patients undergoing IVF (a total of 41 samples were analyzed); receptive endometrium was assessed using the endometrial receptivity array. When bacterial communities from paired endometrial fluid and vaginal aspirate samples within the same subjects were interrogated, different bacterial communities were detected in the uterine cavity and the vagina in some subjects.

Based on its composition, the microbiota in the endometrial fluid, comprising up to 191 operational taxonomic units, was defined as Lactobacillus-dominated microbiota $(>90 \%$ of Lactobacillus spp.) or a non-Lactobacillus-dominated microbiota ( $<90 \%$ Lactobacillus spp. with $>10 \%$ of other bacteria). Although the endometrial microbiota was not hormonally regulated during the acquisition of endometrial receptivity, the presence of a nonLactobacillus-dominated microbiota in a receptive endometrium was associated with a significant decrease in implantation $(60.7 \%$ vs $23.1 \%)$, pregnancy (70.6\% vs $33.3 \%)$, ongoing pregnancy (58.8\% vs $13.3 \%)$, and live birth $(58.8 \%$ vs $6.7 \%)$ rates [11]. Endometrial microbiome in infertile patients was assessed in the study of [12] The endometrial microbiome during the embryo transfer was characterized by analyzing catheter tips with Illumina V4 metagenomics in 70 patients who underwent IVF cycles. Lactobacillus spp. were detected in all 70 samples. Among 70 samples, 33 samples contained over $90 \%$ of Lactobacillus abundance and 50 samples contained over $70 \%$ of Lactobacillus abundance; this finding was consistent with the dominance of Lactobacillus spp. in the lower reproductive tract.

Other vaginal bacteria were also identified from the transfer catheter tips. Corynebacterium spp. were detected in 40 out of 70 patients, Bifidobacterium spp. were detected in 15 out of 70 patients and Staphylococcus spp. were detected in 38 out of 70 patients. Other lactic acid-producing bacteria from the genera Streptococcus spp. were present in 38 out of 70 patients. Thus, our findings suggest the existence of a functional system "microbiota - endometrium", which is characterized by the dominance of Lactobaciluss spp. As a result of various adverse factors, uterine cavity homeostasis may be impaired, which subsequently affects the reproductive outcomes [12].

\section{Conclusion}

The evidence of this study suggests that the uterus is not sterile, the uterine microbiota may differ from the cervical canal microbiota, the uterine microbiota is one of the predictors of the success of embryo implantation.

The study showed that among 33 women who became pregnant, the best results of implantation were observed during the colonization of the uterus only with Lactobacillus spp. both in fresh and frozen-thawed embryo transfers. The overall pregnancy rate was $36.4 \%$ after the colonization of the uterine cavity with association of opportunistic microorganisms with Lactobacillus spp. Provided the sterile uterine cavity, pregnancy occurred in $9.1 \%$ of cases ( $n=3)$. Therefore, the presence of Lactobecillus spp. in the uterus increases the likelihood of pregnancy. At the same time, the high frequency of isolation of Bifidobacterium spp. in non-pregnant women requires clarification of the possible negative effect of these microorganisms on implantation in a larger population of women.

\section{References}

1. Moreno I, Franasiak JM (2017) Endometrial microbiota-new player in town. Fertil Steril 108(1): 32-39. 
2. Jason M Franasiak, Richard T Scott (2017) Endometrial microbiome. Curr Opin Obstet Gynecol 29(3):146-152.

3. Franasiak JM, Werner MD, Juneau CR, Tao X, Landis J, et al. (2016) Endometrial microbiome at the time of embryo transfer: next-generation sequencing of the $16 \mathrm{~S}$ ribosomal subunit. J Assist Reprod Genet 33(1) 129-136.

4. Einenkel R, Zygmunt M, Muzzio Do (2019) Microorganisms in the healthy upper reproductive tract: from denial to beneficial assignments for reproductive biology. Reprod Biol 19(2): 113-118.

5. Lora V Hooper, Dan R Littman, Andrew J Macpherson (2012) Interactions between the microbiota and the immune system. Science 336(6086): 1268-1273.

6. D’Ippolito S, Di Nicuolo F, Pontecorvi A, Gratta M, Scambia G, et al. (2018) Endometrial microbes and microbiome: Recent insights on the inflammatory and immune players of the human endometrium. Am J Reprod Immunol 80(6): e13065.

7. Benner M, Ferwerda G, Joosten I, van der Molen RG (2018) How uterine microbiota might be responsible for a receptive, fertile endometrium.
Hum Reprod Update 24(4): 393-415.

8. Littman DR, Pamer EG (2011) Role of the commensal microbiota in normal and pathogenic host immune responses. Cell Host Microbe 10(4): 311-323

9. Mitchell CM, Haick A, Nkwopara E, Garcia R, Rendi M, et al. (2015) Colonization of the upper genital tract by vaginal bacterial species in nonpregnant women. Am J Obstet Gynecol 212(5):611.

10. Cicineli E, Ballini A, Marinaccio M, Poliseno A, Coscia MF, et al. (2012) Microbiological findings in endometrial specimen: our experience. Arch Gynecol Obstet 285(5): 1325-1329.

11. Moreno I, Codoñer FM, Vilella F, Valbuena D, Martinez-Blanch JF, et al. (2016) Evidence that the endometrial microbiota has an effect on implantation success or failure. Am J Obstet Gynecol 215(6): 684-703.

12. Tao X, Franasiak JM, Zhan Y, Scott RT III, Rajchel J, et al. (2017) Characterizing the endometrial microbiome by analyzing the ultralow bacteria from embryo transfer catheter tips in IVF cycles: next generation sequencing (NGS) analysis of the $16 \mathrm{~S}$ ribosomal gene. Hum Microb J 3: 15-21. 